18-20 December, 2020

Oxford, United Kingdom $11^{\text {th }}$ International Conference on

Humanities, Psychology \& Social Sciences

\title{
The role of politically dissident source characteristics in perceived message reliability
}

Ekström, A.

Lund University

\begin{abstract}
Every day, people chose to trust or distrust pieces of information. Research in epistemology the study and theory of knowledge - has found that both source characteristics and content of a message affect a perceiver's trust in its contents. Despite its relevance to everyday interactions, however, these findings have yet to be extended to politics. Research in social psychology have documented differential treatment of out-group members, including a stronger negative bias toward out-group males than out-group females. In this work, we thus aim to investigate the roles that judge ideology, perceived source ideology and gender of the source person plays in deciding whether to trust message contents. We aim to present participants with juxtapositions of (1) a male or female face (sampled from Chicago faces database, see Ma, Correll, \& Wittenbrink, 2015); (2) a political claim (sampled from political party platforms); and (3) a party affiliation. For each trial, all three variables will be randomized, and participants will be asked to state how much they trust the source of the claim, as well as the claim itself. Participants' ideological convictions will also be assessed. We aim to present preliminary results from this research.
\end{abstract}

Keywords. communication; epistemology; ingroup bias; political psychology; trust 